\title{
Vorbemerkung des Autors und Danksagung
}

Schon mit Beginn meiner Dienstzeit bei Live Oak in der ersten Hälfte der siebziger Jahre hat diese politisch-militärische Organisation mein besonderes Interesse gefunden. Durch meine vierjährige Tätigkeit als Planungsstabsoffizier im International Military Staff (IMS) der NATO gewann ich dann neue Einsichten in das wichtige Thema der Krisenvorsorge und -bewältigung allgemein und zu Berlin im Besonderen.

Während meiner aktiven Dienstzeit habe ich mich dann intensiv mit Fragen der Sicherheit Berlins und mit dem Freien Zugang zur Stadt befasst. Allerdings gab es insbesondere hinsichtlich der "Berlin Contingencies« - der Eventualfallplanungen im Zusammenhang mit Berlin - noch sehr wenig ergiebiges Material. Das hatte mit der großen Sensitivität des Themas und der hohen Geheimhaltungsstufe zu tun. Gegen Ende des Kalten Krieges erschienen vermehrt Arbeiten über die verschiedenen Berlin-Krisen. Die meisten dieser Publikationen litten allerdings darunter, dass die wichtigsten Dokumente in der Regel noch nicht freigegeben waren. Sehr früh schon begann ich auch ein persönliches Tagebuch zu führen, woraus ich für diese Arbeit immer wieder schöpfen konnte.

Nach meinem Abschied aus dem aktiven Dienst im Jahre 1994 erhielt ich Kenntnis, dass die Akten des Stabes Live Oak durch gemeinsamen Beschluss der Vier Mächte im Bundesarchiv, Abteilung Militärarchiv (BArch) in Freiburg im Breisgau als Bestand BW 71 gelagert worden waren. Im Herbst 2005 wurden die Unterlagen dann mit wenigen Ausnahmen automatisch offengelegt. Die folgende Arbeit basiert in erheblichem Maße auf diesem Material.

Die Arbeit soll die Bedeutung von Live Oak als Organisation der politischen Krisenvorsorge und -bewältigung untersuchen und sie einer kritischen Analyse unterziehen. Schwerpunkt der Studie ist die militärische Krisenvorsorge. Die politische Krisenbewältigung ${ }^{1}$ wurde nur insoweit behandelt, als es für die Arbeit notwendig und mit Blick auf die Quellenlage möglich war. Ohne Zweifel wäre diese Aufgabe lohnend, besonders dann, wenn die Bestände im Politischen Archiv des Auswärtigen Amtes (AA), im Bundeskanzleramt (BKA), im Bundesministerium der Verteidigung (BMVg) und in anderen Dienststellen genutzt würden, sofern offengelegt. Dadurch könnten sich Korrekturen, Ergänzungen und neue Erkenntnisse auch für diese Arbeit ergeben.

Es ist klar, dass die Vorteile, die meine berufliche Beschäftigung in Kombination mit dem mir zugänglichen Aktenmaterial und den daraus erwachsenden Synergieeffekten

1 Dazu gibt es inzwischen eine Anzahl an Studien, die allerdings den militärischen Anteil meist eher kursorisch behandeln. Eine Auswahl der hier verwendeten Literatur findet sich im Literaturverzeichnis. 
bringen, durch fortdauernde Reflexion über den Status als historisch Handelnder erkauft werden müssen. Zeitzeugen sind in der Forschung - zu Recht - immer Quellen, die besonders kritisch betrachtet werden. Für mich, der ich über ein historisches Thema berichte, in dem ich selbst tätig war, bedeutet dies besondere Vorsicht im Umgang mit den Akten und den persönlichen Erinnerungen.

Die Leser möchte ich bereits jetzt um Nachsicht dafür bitten, dass die Vielzahl der im Folgenden immer wieder angesprochenen Dienststellen, Behörden, Organisationen usw. zwangsläufig zur ausgedehnten Verwendung von Abkürzungen aus mehreren Sprachen führt. Es ist meine Hoffnung, dass sie im Laufe der Lektüre dem Leser geläufig werden; wenn nicht, möge das umfangreiche Abkürzungsverzeichnis weiterhelfen.

Die schriftlichen Quellen dieser Untersuchung sind überwiegend in englischer Sprache verfasst. Für manche englische Fachbegriffe existieren gängige deutsche Entsprechungen, für einige andere habe ich deutsche Übersetzungen eingeführt. Nicht immer wollen sich aber befriedigende deutsche Begriffe finden lassen, weswegen es gelegentlich auch zu Missverständnissen kommen kann. Das trifft vor allem auf das unscheinbare, aber in unserem Zusammenhang wichtige englische Wörtchen "probe« zu. Wird es im Satzzusammenhang groß geschrieben, ist seine englischsprachige Herkunft und Verwendung nicht ohne Weiteres erkennbar und der Leser wundert sich vielleicht über Ausdrücke wie Autobahn-»Probe«. Gemeint sind Sondierungsfahrten zur Auslotung der Reaktion von DDR bzw. Sowjetunion auf westalliierte Transporte auf dem Landweg nach oder von West-Berlin. Noch weitere deutsch-englische Sprachmischungen werden dem Leser begegnen - manchmal vielleicht durch Nachlässigkeit begünstigt, gewiss aber auch ein Nachklang jener intensiven historischen Verflechtung von westalliierten und deutschen Bemühungen im Zusammenhang mit der Aufrechterhaltung der Verbindungswege nach Berlin - das große Thema der vorliegenden Arbeit.

In Quellenzitaten taucht die ehemalige ostdeutsche Republik gelegentlich in Anführungsstrichen auf: „DDR«. In diesen Fällen handelt es sich nicht um Sonderheiten des Autors, sondern um sprachliche Relikte aus dem Kalten Krieg. Sprachliche Zwangskorsette rund um die Schreibweisen der geteilten Stadt - Ost-Berlin, Ostberlin oder Berlin (Ost), adäquat West-Berlin, Westberlin oder Berlin (West) - und ähnliches mehr scheinen mir überholt, sodass ich um Verständnis für einen möglicherweise generösen Umgang mit ehemals erbittert gehandhabten Sprachregelungen und das Nebeneinander verschiedener Formen in Darstellung und Quellen bitte.

Ein Glossar im Anhang bietet dem Leser Definitionen von vorwiegend in diesem $\mathrm{Zu}$ sammenhang gebrauchten Fachtermini. Im Anhang findet sich auch eine Übersicht der im Text häufig verwendeten Decknamen für Operationspläne und Übungen. Zahlreiche Karten und Grafiken helfen zudem, komplexe Zusammenhänge besser zu verstehen. Ein Personenregister beschließt den Band.

Das vorliegende Buch über die Geschichte von Live Oak hat sich im Verlauf der Arbeit als ein Großprojekt erwiesen. Am Ende waren es - mit Unterbrechungen - zehn Jahre der Recherche, der Akteneinsicht, der fachlichen Auseinandersetzung mit Historikern und Zeitzeugen und schließlich des Schreibens. Hinzu kamen noch die Monate der 
„Veredelung“ des Manuskriptes: das Zeichnen der Karten, das Lektorat und das Setzen des Buches. Es gehört zu den schönen Arbeiten am Ende eines Projektes, nunmehr jenen Menschen Dank auszusprechen, die am Entstehen dieses Werkes beteiligt waren oder "Mitschuld" an seinem Zustandekommen tragen.

Zunächst habe ich Frau Prof. Dr. Beatrice Heuser zu danken, die als damalige Leiterin der Abteilung Forschung des Militärgeschichtlichen Forschungsamtes (MGFA) meinem Antrag, eine Studie über Krisenmanagement im Kalten Krieg am Beispiel von Live Oak zu schreiben, sofortige Unterstützung zusagte. Ungemein wertvolle und fast tägliche Hilfe erhielt ich im Bundesarchiv, Abteilung Militärarchiv, in Freiburg im Breisgau. Dafür sorgten vor allem die zuständigen Referatsleiter, Dr. Volker Gießler und Dr. Andreas Kunz. Besonders hervorzuheben gilt es hier natürlich die Mitarbeiter des Magazindienstes. Die für die Offenlegung vieler Korrespondenz- und Gegenüberlieferungen zuständige Gruppe, zunächst unter Major Dr. Klaus Storkmann (heute Oberstleutnant), dann unter Oberstleutnant Michael Peter, ermöglichte vergleichende Recherchen. Ihnen allen bin ich für ihre Arbeit von Herzen dankbar. Dass mein Tun dann positiv gewürdigt wurde, dafür danke ich Herrn Oberst i.G. Dr. Burkhard Köster (damals MGFA/ZMSBw) und Oberst a.D. Dr. Norbert Wiggershaus ( $\dagger$ ). Durch seine Entscheidung, das Ergebnis meiner Recherchen im ZMSBw zu publizieren, erkannte Prof. Dr. Michael Epkenhans mein Werk als wichtig an, wofür ich ihm sehr verbunden bin.

Dem ehemaligen Botschafter der Bundesrepublik und dann Präsidenten des BND, Dr. Hans-Georg Wieck, bin ich zu besonderem Dank verpflichtet, weil er, auch als Zeitzeuge und Diplomat, wiederholt schriftlich und mündlich zu allgemein politischen und zu speziellen, die Berlinproblematik betreffenden Fragen Stellung nahm, was die Arbeit wesentlich befruchtete. Auch zu einem ausführlichen Interview stand er mir als exzellenter Kenner der deutschen Außen- und Sicherheitspolitik zur Verfügung.

Meinen beiden Kameraden der "Air Section" und des NAVCORCENT bei Live Oak, Oberst a.D. Christian Hartig und Fregattenkapitän a.D. Bernd Basche, danke ich für die kritische Durchsicht der ihre jeweilige Teilstreitkraft behandelnden Kapitel. Bernd Basche leistete auch als geborener und kenntnisreicher "Berliner Jung" " unverzichtbare Dienste bei meinen Ausführungen zur problembelasteten Lage der geteilten Stadt Berlin.

Der langjährige SHAPE-Historian Dr. Gregory Pedlow hat dankenswerterweise durch seine intime Kenntnis der Akten der NATO, von SHAPE und Live Oak viele Fragen ausführlich beantwortet, oft weit über die Aktenlage hinausreichend.

Dass aus dem Text schließlich ein Buch wurde, ist dem Team des Fachbereichs Publikationen im ZMSBw unter Leitung von Dr. Christian Adam geschuldet. Carola Klinke war zuständig für den Satz des Buches und für die technische Aufbereitung der Abbildungen. Frank Schemmerling hat die aufwändigen, eindrucksvollen Karten und Grafiken erstellt, und Michael Thomae schließlich hat das arbeitsreiche Lektorat übernommen. Ihnen allen bin ich zu großem Dank verpflichtet. Danken möchte ich in diesem Zusammenhang auch Dr. Arnim Lang (ehemals Forschungsbereich »Militärgeschichte nach 1945« am ZMSBw) für seinen »neutralen Blick» auf alles.

In zwei weiteren Archiven fand ich gute Aufnahme und Unterstützung in der Suche nach relevantem Material. So danke ich Dr. Philipp von Boeselager und Herrn Knud Pillich im Politischen Archiv des Auswärtigen Amtes (PA AA) sowie Frau Irene Schaarschmidt bei der Bundesbehörde für Stasi-Unterlagen (BStU), beide in Berlin. 
Im Deutschen Historischen Institut Moskau hat mir Dr. Mathias Uhl durch aktuelle Hinweise die Augen für leider nur wenige, aber doch wichtige Quellen aus sowjetischen und russischen Archiven geöffnet, wofür ich ihm zu Dank verpflichtet bin.

Herrn Oberstleutnant Nils Windhäuser, damals in SHAPE, danke ich für das Herstellen einer Verbindung zum dortigen Fotoarchiv. Durch den Kontakt zur Familie des ersten deutschen Verbindungsoffiziers bei Live Oak, Brigadegeneral Wilhelm Thomas, war es dankenswerterweise möglich, an seltenes Fotomaterial aus Privatbesitz zu gelangen, das zum Teil auch für dieses Buch genutzt wurde.

Unentwegte Unterstützung fand ich in der Außenstelle des ZMSBw im 9. Stockwerk des Bundesarchiv-Militärarchivs durch den ıguten Geist‘ Frau Cynthia Flohr, einer Kennerin aller Wege und Möglichkeiten des Hauses; durch Herrn Albrecht Kästner zu allen Fragen die NVA betreffend, sowie bei meinem Schreibtisch gegenüber, Herrn Dr. Alexander Jaser, nicht zu vergessen die Herren Ulf Balke und Dr. Jürgen Förster, die meine Arbeit über viele Jahre fruchtbar begleiteten.

Schließlich bin ich meiner Frau für ihre unendliche Geduld - mehr als ein Jahrzehnt! - und ihre nie versiegende, feste Hoffnung auf einen guten Schluss unendlich dankbar.

Harald van Nes 